\title{
An Improved Time-Domain Damage Detection Method for Railway Bridges Subjected to Unknown Moving Loads
}

\author{
Mahdi Shahbaznia', Morteza Raissi Dehkordi* ${ }^{*}$ Akbar Mirzaee \\ ${ }^{1}$ School of Civil Engineering, Iran University of Science and Technology, Narmak, Tehran 16846-13114, Iran \\ * Corresponding author, e-mail: mraissi@iust.ac.ir
}

Received: 26 February 2020, Accepted: 27 May 2020, Published online: 13 July 2020

\begin{abstract}
There is considerable interest in structural health monitoring (SHM) and damage detection of bridges and considerable progress has been made in this field in recent years. However, several challenges such as sensitivity to low levels of damage and identification without the knowledge of the moving load remain and need to be precisely investigated by researchers. The current work addresses such challenges and proposes an efficient response sensitivity-based model updating procedure in time-domain for damage identification of railway bridges subjected to unknown moving loads. The bridge is modelled as an Euler-Bernoulli beam and the train is modelled as a set of sprung masses passing over the beam. Structural damage is considered as a reduction in the modulus of elasticity of the elements. Sensitivity analysis and Tikhonov regularization methods are adopted and used to solve the inverse problem of the model updating. To verify the efficiency of the model, two numerical models with multiple damage scenarios subjected to unknown moving loads are analyzed. In addition, the efficiency of the proposed method in the presence of measurement noise is also verified. Numerical results reveal that the proposed model-updating procedure simultaneously identifies structural damages as well as the unknown moving loads with an acceptable accuracy. The effect of critical parameters such as mass and speed of the moving vehicle on the accuracy of identification results is investigated as well. Based on the findings of this research, the proposed method can be adopted and applied to online and long-term health monitoring of real bridge structures.
\end{abstract}

Keywords

railway bridge, model-updating, damage detection, sensitivity analysis, unknown input, time-domain

\section{Introduction}

Structural Health Monitoring (SHM) has been emerged as a robust technology for long-term monitoring of infrastructures such as bridges, tall buildings, dams, aerospace structures, and so forth. Damage detection algorithms are part of the SHM process which aim to locate and quantify the occurred damages. Numerous works in the frequency-domain have been performed on the detection of local damages in structures using changes in modal properties such as natural frequency, mode shape, mode shape curvature and so on [1]. Implementing frequency-domain techniques for practical damage detection of bridges under operation encounters several problems. Firstly, the bridge-vehicle interaction model constructs a coupled equation of motion with time-variant elements. Therefore, using frequency-domain techniques does not take into account the interaction of the vehicle-bridge system. Secondly, the modal frequencies of these bridge infrastructures are very high and it is almost impossible to observe these high frequencies. Last but not least, the modal properties are strongly affected by environmental parameters such as temperature, temperature gradient, humidity, and so on. It is indispensable to take the above-mentioned parameters into consideration and eliminate environmental components during identification process [2].

Based on the aforementioned reasons, to carry out an effective damage identification of bridges, the analysis is performed in the time-domain by the direct comparison of the simulated as well as measured responses. A time-domain formulation has been proposed so as to detect damages in a beam utilizing data originating from the linear beam oscillator dynamic interaction [3].

Model-updating procedures are the optimization methods whose objective functions minimize the difference between recorded response of the measured structure and 
that of analytical model [4]. The procedure applies a sensitivity-based change to stiffness parameters in such a way that best reflects measured responses. One challenge concerning this method is to select the right vibration parameter for updating the FE model. For short-span railway bridges it has been demonstrated that bridge displacement response is more appropriate than that of acceleration so as to update the railway bridge stiffness [5], whereas other researchers observed that acceleration time-history of the structure presents more exhaustive and useful information which can be effectively used for damage identification of structures. The performance of finite element (FE) model updating to identify the induced damage in a two-story reinforced concrete masonry-infilled building is investigated using vibration data as well as lidar (light detection and ranging) scans [6].

One important aspect of a SHM system is related to obtained measurement data. It is well known that poorly distributed sensors are not capable of capturing crucial characteristics of the structure. A mode shape sensitivity method is presented for optimal sensor placement (OSP) [7].

Simultaneous identification techniques are the methods eliciting structural parameters based on the measured responses without the knowledge of the moving loads or any external excitations [8]. In fact, the identification process simultaneously determines the amount and location of structural damages along with the values of the moving load. Adjoint method is proposed for identification of structural damage in structures subjected to unknown moving masses [9].

A number of optimization algorithms have been proposed to tackle the weaknesses of searching algorithms in model updating for damage detection problems [10, 11]. In Contrast to general optimization algorithms, sensitivity-based methods have revealed their efficiency in structural damage detection problems. A robust sensitivity-based approach has been proposed to identify the local damages in a structure directly from the measured dynamic responses [12]. An improved sensitivity-based damage identification approach has also been presented for bridge structures subjected to moving loads without the knowledge of the vehicle properties [13].

Damage and load identification problems are considered as inverse problems, and their numerical ill-conditioning seems to be the main factor influencing the accuracy of the identification results. The accuracy can be improved by regularization methods. Based on the context of the inverse problem which might be over-determined or under-determined, a number of optimization algorithms are presented and evaluated for optimal solution of the inverse problem.

In frequency-domain damage detection algorithms, dealing basically with modal characteristics of the structure, $\ell 1$-norm minimization or $\ell 1$ regularization has been successfully applied [14]. A sparse damage detection technique is developed using merely the first few frequencies. The technique utilizes the capability of $\ell 2$ regularization so as to reconstruct sparse damage among a large number of elements of the entire structure [15]. Recently two sparsity constrained optimization algorithms are employed and compared for damage identification: $\ell 1$-norm minimization and non-negative least square (NNLS) solution [7]. It was demonstrated that NNLS shows a better performance in damage identification than $\ell 1$-norm.

Time-domain damage detection algorithms which deal with time history of the vibrational response, are generally over-determined inverse problems. A standard technique to prevent over-determinacy is to use $\ell 2$-norm minimization or Tikhonov regularization. In the context of finite element model updating in structural dynamics $\ell 2$-norm regularization has been successfully employed [16]. An adaptive regularization approach has been presented for solving the nonlinear model updating inverse problems [17].

In this research project an improved model-updating identification method in the time-domain is proposed to identify structural damages subjected to unknown moving load. The proposed method consists of two iterative steps employed to evaluate the moving load as well as structural damages. In the current research work, the bridge is modelled as an Euler-Bernoulli beam subjected to unknown moving load. The damage is introduced as a reduction in stiffness of structural elements according to previous works in this respect $[18,19]$. Moving load is simulated as a set of sprung-masses passing over the beam with constant velocity and unknown variable weights. The coupled equation of motion of the bridge-vehicle system is derived and force and stiffness sensitivity matrices were calculated using the direct differential method (DDM) and are introduced as three dimensional matrices in time-domain. Tikhonov regularization using L-Curve method is applied to solve the ill-posed inverse problem of damage detection.

In recent years, considerable progress has been made in the field of SHM and damage detection of bridges. However, several challenges such as unknown moving load, sensitivity to low levels of damage and identification 
with limited number of sensors remain and need to be precisely investigated by researchers. The proposed FE model-updating method addresses such challenges and is merely based on the vibrational response. Numerical results prove that the proposed method is capable of accurately detecting structural damages in the presence of unknown moving loads. It is also observed that simulating the train in the form of sprung-masses guaranties the interaction of bridge-vehicle system and dramatically increases the accuracy of the identification compared to existing research works in this respect. To simulate real conditions, capability of the model for simultaneous identification of bridge structure in the presence of measurement noise is investigated by adding a noise to the measured data as well. In addition, the effect of speed and mass of the moving vehicle on the accuracy of the identification results is investigated as well and an appropriate range for these critical parameters is suggested. Considering these qualifications, the proposed method could be effectively adopted and applied for continuous and online monitoring of railway bridges.

\section{Formulation}

In this section, the equations of motion of train and bridge are derived and coupled so as to obtain the equation of motion governing bridge-vehicle system. The resulting equation can be solved in time-domain using direct time integral methods such as Newmark- $\beta$.

\subsection{Sprung mass model of bridge and train system}

Most of the recent studies have considered the vehicle as a single moving load which ignore the suspension properties of the moving vehicle. In the current survey the train is modelled as a set of sprung-masses moving over the beam with constant velocity. The sprung-mass modelling has the following merits:

1. The suspension properties of the moving vehicle are included in the equation of motion.

2. Elements of the bridge-vehicle equation of motion are time-variant which guarantees the proper consideration of bridge-vehicle interaction.

To construct the equation of motion, both equations of train and bridge should be formulated. As shown in Fig. 1, the train is modeled schematically as $N_{v}$ number of sprungmasses with distance $D_{j}$ over a continuous beam, where $u_{w j}$ and $u_{v j}$ denote vertical displacements of $j^{\text {th }}$ wheel set and vehicle and $u_{b}(x . t)$ is vertical displacement of the beam at distance $(x)$ and time $(t)$.

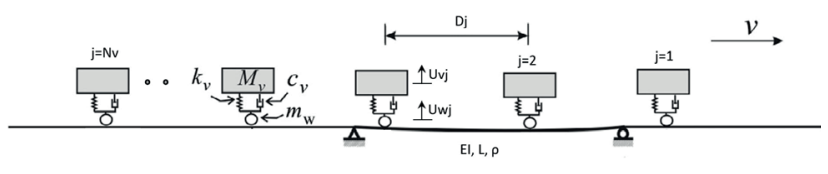

Fig. 1 Sprung-Mass model of the bridge-vehicle system

For the $\mathrm{j}^{\text {th }}$ one-foot sprung mass, the upper mass, $m_{v j}$, is part of the vehicle mass and the lower mass, $m_{w j}$, represents the wheel set; the suspension system possesses stiffness value of $K_{v j}$ and damping value of $C_{v j}$. The vertical displacement of $j^{\text {th }}$ wheel set and the upper mass are denoted by $\mathbf{U w j}$ and $\mathbf{U v j}$, respectively. The distance between the $j^{\text {th }}$ and $j+1^{\text {th }}$ one-foot sprung mass is introduced by $D_{j}$.

It has been indicated that the contact force between the bridge and the lower mass is $f_{c j}$, which consists of a static element, $f_{w j}$, because of weight and a dynamic element, $f_{d j}$, due to vertical vibration [20] as described in Eqs. (1-2):

$f_{c j}=f_{w j}+f_{d j}$,

$f_{w j}=\left(m_{w j}+m_{v j}\right) \times g$.

The equilibrium equation of train due to the vertical force can be written in the form of Eq. (3):

$$
\begin{aligned}
& {\left[\begin{array}{cc}
\mathbf{M}_{\mathbf{w}} & 0 \\
0 & \mathbf{M}_{\mathbf{v}}
\end{array}\right]\left\{\begin{array}{l}
\ddot{\boldsymbol{u}}_{w} \\
\ddot{\boldsymbol{u}}_{v}
\end{array}\right\}+\left[\begin{array}{cc}
\mathbf{C}_{\mathbf{v}} & -\mathbf{C}_{\mathbf{v}} \\
-\mathbf{C}_{\mathbf{v}} & \mathbf{C}_{\mathbf{v}}
\end{array}\right]\left\{\begin{array}{l}
\dot{u}_{w} \\
\dot{\boldsymbol{u}}_{v}
\end{array}\right\}} \\
& +\left[\begin{array}{cc}
\mathbf{K}_{\mathbf{v}} & -\mathbf{K}_{\mathbf{v}} \\
-\mathbf{K}_{\mathbf{v}} & \mathbf{K}_{\mathbf{v}}
\end{array}\right]\left\{\begin{array}{l}
\boldsymbol{u}_{w} \\
\boldsymbol{u}_{v}
\end{array}\right\}=\left\{\begin{array}{l}
f_{d} \\
\{0\}
\end{array}\right\} .
\end{aligned}
$$

The bridge is divided into $N_{e}$ elements with the same length, $L$, that are shorter than the minimum distance between the units of sprung masses. Mass and stiffness matrices of the beam, $\mathbf{M b}$ and $\mathbf{K b}$ are constructed by assembling mass and stiffness matrices of the elements. Bridge damping type is Rayleigh damping with the coefficient $\alpha$ and $\beta$ calculated from Eq. (4):

$\left\{\begin{array}{l}\alpha \\ \beta\end{array}\right\}=\frac{2 \zeta}{w_{1}+w_{2}}\left\{\begin{array}{c}w_{1} \times w_{2} \\ 1\end{array}\right\}$,

where $w_{1}$ and $w_{2}$ are the first and second modal natural frequencies and $\zeta$ is the modal damping ratio. Therefore, the damping matrix of the beam is as follows:

$\mathbf{C}_{\mathbf{b}}=\alpha \mathbf{M}_{\mathbf{b}}+\beta \mathbf{K}_{\mathbf{b}}$.

The bridge is initially in static condition, so when the train approaches the bridge, the external loads acting on the bridge are contact forces, $-\mathbf{N} f \boldsymbol{c}$, between the wheel and bridge, where $\mathbf{N}$ is the mapping matrix that utilizes cubic Hermit interpolation functions. The equation of motion of the whole bridge can be assembled as Eq. (6): 


$$
\mathbf{M}_{\mathbf{b}} \ddot{u}_{b}+\mathbf{C}_{\mathbf{b}} \dot{u}_{b}+\mathbf{K}_{\mathbf{b}} \boldsymbol{u}_{b}=-\mathbf{N} f_{c}
$$

The Eqs. (3) and (6) need to be coupled to construct the equation of motion of bridge-vehicle system. The above-mentioned equations consist of three variables, $\mathbf{u}_{\mathbf{w}}, \mathbf{u}_{\mathbf{v}}$ and $\mathbf{u}_{\mathbf{b}}$. The coupling of the mentioned equations eliminates the vibrations of wheel sets, $\mathbf{u}_{\mathbf{w}}, \dot{\mathbf{u}}_{\mathbf{w}}$ and $\ddot{\mathbf{u}}_{\mathbf{w}}$. It is assumed that the wheel set is always in contact with the bridge, so the vertical vibrations of wheel sets can be substituted by the bridge nodal displacements as Eqs. (7-9), Where $\mathbf{V}$ and $\mathbf{A}$ are velocity and acceleration of train, respectively. $\mathbf{u}_{\mathbf{w}}$ and $\mathbf{u}_{\mathbf{b}}$ are the responses of the wheel sets and the beam, respectively.

$$
\begin{aligned}
& \boldsymbol{u}_{\boldsymbol{w}}=\mathbf{N}^{T} \boldsymbol{u}_{\boldsymbol{b}} \\
& \dot{\boldsymbol{u}}_{\boldsymbol{w}}=\mathbf{V} \mathbf{N}_{, \mathbf{x}}^{T} \boldsymbol{u}_{\boldsymbol{b}}+\mathbf{N}^{T} \dot{\boldsymbol{u}}_{\boldsymbol{b}} \\
& \ddot{\boldsymbol{u}}_{\boldsymbol{w}}=\left(\mathbf{A} \mathbf{N}_{, \mathbf{x}}{ }^{T}+\mathbf{V}^{2} \mathbf{N}_{, \mathbf{x x}}{ }^{T}\right) \boldsymbol{u}_{\boldsymbol{b}}+2 \mathbf{V} \mathbf{N}_{, \mathbf{x}}{ }^{T} \dot{\boldsymbol{u}}_{\boldsymbol{b}}+\mathbf{N}^{T} \ddot{\boldsymbol{u}}_{\boldsymbol{b}}
\end{aligned}
$$

Substituting Eqs. (7-9) into the first row of Eq. (3) results in the expression of dynamic contact forces $\mathbf{f}_{\mathbf{d}}$ as Eq. (10), in which vibrations of the wheel sets are eliminated from the equation.

$$
\begin{aligned}
& \boldsymbol{f}_{\boldsymbol{d}}=\left(\mathbf{M}_{\mathbf{w}} \mathbf{A} \mathbf{N}_{, \mathbf{x}}{ }^{T}+\mathbf{M}_{\mathbf{w}} \mathbf{V}^{2} \mathbf{N}_{, \mathbf{x x}}{ }^{T}+\mathbf{C}_{\mathbf{v}} \mathbf{V} \mathbf{N}_{, \mathbf{x}}{ }^{T}+\mathbf{K}_{\mathbf{v}} \mathbf{N}^{T}\right) \boldsymbol{u}_{\boldsymbol{b}} \\
& +\left(2 \mathbf{M}_{\mathbf{w}} \mathbf{V N}_{, \mathbf{x}}{ }^{T}+\mathbf{C}_{\mathbf{v}} \mathbf{N}^{\mathbf{T}}\right) \dot{\boldsymbol{u}}_{\boldsymbol{b}}+\mathbf{M}_{\mathbf{W}} \mathbf{N}^{T} \ddot{\boldsymbol{u}}_{\boldsymbol{b}}-\mathbf{C}_{\mathbf{v}} \dot{\boldsymbol{u}}_{\boldsymbol{v}}-\mathbf{K}_{\mathbf{v}} \boldsymbol{u}_{\boldsymbol{v}}
\end{aligned}
$$

Using Eqs. (1) and (10), new form of Eq. 6 will be derived as Eq. (11) which is equation of motion of the bridge:

$$
\mathbf{M}_{\mathrm{b}} \ddot{u}_{b}+\mathbf{C}_{\mathrm{b}} \dot{u}_{b}+\mathbf{K}_{\mathrm{b}} \boldsymbol{u}_{b}+\mathbf{N} f_{d}=-\mathbf{N} f_{w}
$$

To construct equation of motion of the upper mass, $\mathbf{u}_{\mathbf{v}}$, Eqs. (7-9) are substituted into the second row of Eq. (3) The result is as follows:

$\mathbf{M}_{\mathbf{v}} \ddot{\boldsymbol{u}}_{\boldsymbol{v}}+\mathbf{C}_{\mathbf{v}} \dot{\boldsymbol{u}}_{\boldsymbol{v}}+\mathbf{K}_{\mathrm{v}} \boldsymbol{u}_{\boldsymbol{v}}=\left(\mathbf{C}_{\mathbf{v}} \mathbf{V N} \mathbf{N}_{, \mathbf{x}}{ }^{T}+\mathbf{K}_{\mathbf{v}} \mathbf{N}^{T}\right) \boldsymbol{u}_{\boldsymbol{b}}+\mathbf{C}_{\mathbf{v}} \mathbf{N}^{T} \dot{\boldsymbol{u}}_{\boldsymbol{b}}$

Finally, writing Eqs. (11-12) in the form of matrix will result in the coupled equation of motion of the bridge and train as follows:

$$
\begin{aligned}
& {\left[\begin{array}{cc}
\mathbf{M}_{11} & 0 \\
0 & \mathbf{M}_{22}
\end{array}\right]\left\{\begin{array}{l}
\ddot{\boldsymbol{u}}_{\boldsymbol{b}} \\
\ddot{\boldsymbol{u}}_{\boldsymbol{v}}
\end{array}\right\}+\left[\begin{array}{ll}
\mathbf{C}_{11} & \mathbf{C}_{12} \\
\mathbf{C}_{21} & \mathbf{C}_{22}
\end{array}\right]\left\{\begin{array}{l}
\dot{\boldsymbol{u}}_{\boldsymbol{b}} \\
\dot{\boldsymbol{u}}_{\boldsymbol{v}}
\end{array}\right\}} \\
& +\left[\begin{array}{ll}
\mathbf{K}_{11} & \mathbf{K}_{12} \\
\mathbf{K}_{21} & \mathbf{K}_{22}
\end{array}\right]\left\{\begin{array}{l}
\boldsymbol{u}_{\boldsymbol{b}} \\
\boldsymbol{u}_{\boldsymbol{v}}
\end{array}\right\}=\left\{\begin{array}{c}
\boldsymbol{P}_{\boldsymbol{b}} \\
\{0\}
\end{array}\right\} .
\end{aligned}
$$

To simply express the equation of motion, the short form of Eq. (13) is written as Eq. (14):
$\mathbf{M} \ddot{\boldsymbol{u}}+\mathbf{C} \dot{\boldsymbol{u}}+\mathbf{K} \boldsymbol{u}=\boldsymbol{P}(t)$

where:

$$
\begin{aligned}
& \mathbf{M}_{11}=\mathbf{M}_{\mathbf{b}}+\mathbf{N} \mathbf{M}_{\mathbf{w}} \mathbf{N}^{T} \\
& \mathbf{M}_{22}=\mathbf{M}_{\mathbf{v}} \\
& \mathbf{C}_{11}=\mathbf{C}_{\mathbf{b}}+2 \mathbf{N M}_{\mathbf{w}} \mathbf{V} \mathbf{N}_{, \mathbf{x}}{ }^{T}+\mathbf{N C}_{\mathbf{v}} \mathbf{N}^{T} \\
& \mathbf{C}_{12}=-\mathbf{N C}_{\mathbf{v}} \\
& \mathbf{C}_{21}=-\mathbf{C}_{\mathbf{v}} \mathbf{N}^{T} \\
& \mathbf{C}_{22}=\mathbf{C}_{\mathbf{v}} \\
& \mathbf{K}_{11}=\mathbf{K}_{\mathbf{b}}+\mathbf{N M}_{\mathbf{w}} \mathbf{A} \mathbf{N}_{, \mathbf{x}}{ }^{T}+\mathbf{N M}_{\mathbf{w}} \mathbf{V}^{2} \mathbf{N}_{, \mathbf{x x}}{ }^{T} \\
& +\mathbf{N C}_{\mathbf{v}} \mathbf{V} \mathbf{N}_{, \mathbf{x}}{ }^{T}+\mathbf{N K}_{\mathbf{v}} \mathbf{N}^{T} \\
& \mathbf{K}_{12}=-\mathbf{N K}_{\mathbf{v}} \\
& \mathbf{K}_{21}=-\mathbf{C}_{\mathbf{v}} \mathbf{V} \mathbf{N}_{, \mathbf{x}}{ }^{T}-\mathbf{K}_{\mathbf{v}} \mathbf{N}^{\mathbf{T}} \\
& \mathbf{K}_{22}=\mathbf{K}_{\mathbf{v}} \\
& \left\{\boldsymbol{P}_{\boldsymbol{b}}\right\}=-\mathbf{N} f_{w}
\end{aligned}
$$

Equation (14) can be solved through direct time integral methods such as Newmark- $\beta$. This method has two different approaches, namely constant average acceleration and linear acceleration. Constant average acceleration with coefficients $\left(\gamma=\frac{1}{2}, \beta=\frac{1}{4}\right)$ is stable for small amounts of time step, $\Delta t$ [21]. Therefore, in this research project, Newmark- $\beta$ with constant average acceleration is employed to solve the equation of motion.

\subsection{Solution of the inverse problem}

When the parameters of a model are unknown, they need to be estimated using measured data. The measured response is a non-linear function of the parameters. Therefore, minimizing the error between the measured and predicted responses will yield a non-linear optimization problem. Therefore, the identification problem can be expressed as follows so as to find the perturbation vector $\delta \boldsymbol{\alpha}$ such that the calculated response best matches the measured response, i.e. :

$\delta z=\mathbf{S} \delta \alpha$,

$\delta z=\ddot{u}_{m}-\ddot{u}_{c a l}$, 
where $\{\delta z\}$ is the error vector in the measured output, $\ddot{\mathbf{u}}_{\mathbf{m}}$ is the acceleration response of damaged model as target of the identification process, and $\ddot{\mathbf{u}}_{\text {cal }}$ is the calculated acceleration response of updating model. $\mathbf{S}$ is two-dimensional sensitivity matrix whose elements are adopted from the previously proposed three dimensional sensitivity matrix [12].

$\delta \alpha=\mathbf{S}^{\mathbf{T}} \mathbf{S}^{-1} \mathbf{S}^{\mathbf{T}} \delta z$

$\alpha(j+1)=\alpha(j)+\mathbf{S}_{\mathbf{j}}^{\mathbf{T}} \mathbf{S}_{\mathbf{j}}{ }^{-1} \mathbf{S}_{\mathbf{j}}^{\mathbf{T}} \boldsymbol{\delta} \boldsymbol{z}$

$\boldsymbol{\delta} \boldsymbol{\alpha}$ is the perturbation which improves the physical parameter $\boldsymbol{\alpha}$ in each iteration. The subscript $j$ indicates the iteration number by which the sensitivity matrix is computed. As shown in Fig. 1 the iterative procedure is continued to come up with the determined tolerance.

Equation (28) can be solved using simple least squares method, but it has been realized that Tikhonov regularization is an optimal solution [17]. After regularization, the Eq. (28) can be written in the form of Eq. (30):

$\boldsymbol{\delta} \boldsymbol{\alpha}=\left(\mathbf{S}^{\mathbf{T}} \mathbf{S}+\lambda_{R} \mathbf{I}\right)^{-1} \mathbf{S}^{\mathbf{T}} \boldsymbol{\delta} \boldsymbol{z}$,

where $\lambda_{R}$ is a regularization parameter, as a non-negative damping coefficient governing the participation of leastsquares error in the solution. Based on the findings of previous researchers, in this research, Tikhonov regularization with L-curve method is used to solve the ill-posed inverse problem [22].

\subsection{Finite element model-updating procedure for damage detection}

FE model updating in structural dynamics is the process of updating the initial model of structure so that it can better reproduce the measured responses of the actual structure. The updated model can be used to identify and locate structural damages occurred in infrastructures during years of serviceability. As illustrated in Fig. 2 the iteration process consists of two main iterative procedures: The first iteration is employed for force identification, while the second iteration is employed for physical parameter identification using identified force. Where: $\boldsymbol{E}_{\boldsymbol{u}}, \boldsymbol{E}_{\boldsymbol{d}}$ and $\boldsymbol{E}_{\boldsymbol{n}}$ are modulus of elasticity for undamaged, damaged and updating models respectively, $\boldsymbol{P}, \boldsymbol{P}_{\mathbf{0}}$ and $\boldsymbol{P}_{\boldsymbol{n}}$ are actual, initial guess and updated values for the moving load respectively, SF and SK are sensitivity matrices due to force and physical parameters respectively, $\boldsymbol{R}_{\boldsymbol{d}}$ and $\boldsymbol{R}_{\boldsymbol{n}}$ are responses of the damaged and updating models respectively, $\boldsymbol{d P}$ and $\boldsymbol{d} \boldsymbol{E}$ are perturbation due to load and physical parameters respectively. Tolerance is set as $1 \times 10^{-4}$.

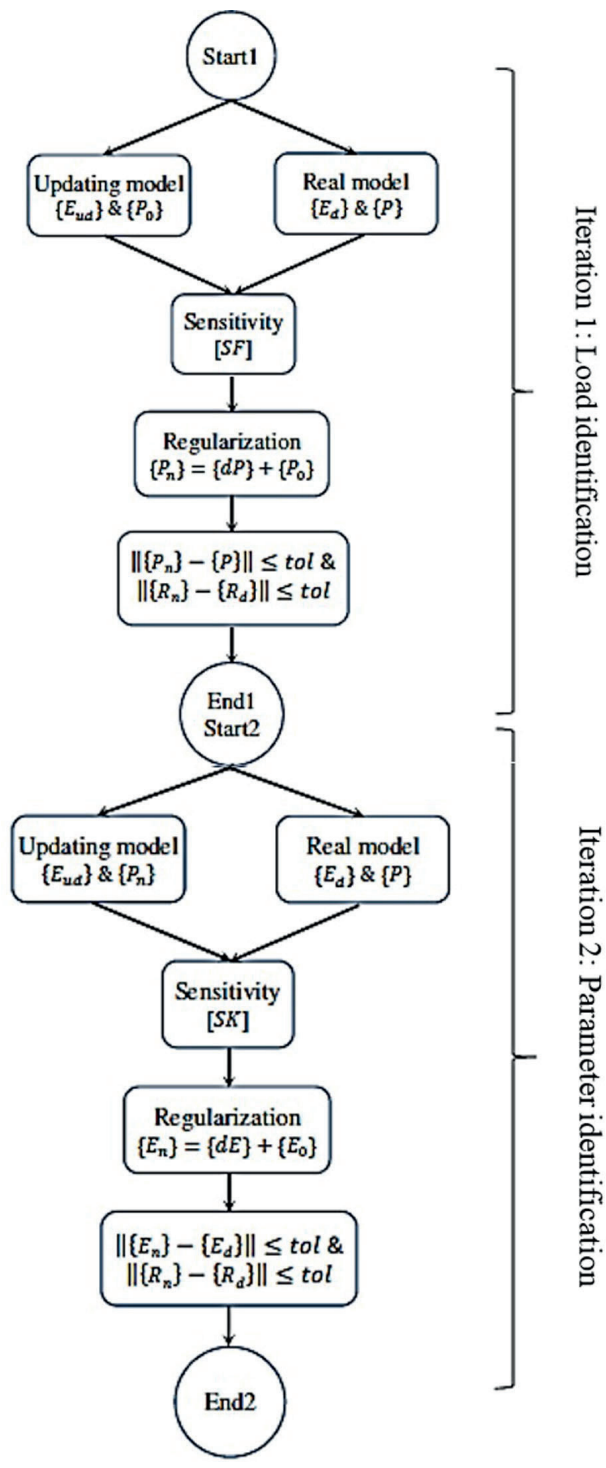

Fig. 2 FE model-updating procedure for simultaneous identification

This approach minimizes the difference between acceleration response time-history of the measured and analytical models. This problem may be expressed as the minimization of $\boldsymbol{\delta} \boldsymbol{z}$ in Eq. (31),

$\delta z=\ddot{u}_{m}-\ddot{u}_{c a l}$,

where $\boldsymbol{\delta} \boldsymbol{z}$ is the error vector in the measured output and $\ddot{\boldsymbol{u}}_{\boldsymbol{m}}$ and $\ddot{\boldsymbol{u}}_{\text {cal }}$ are the measured and calculated acceleration response vectors, respectively. In the inverse problem of damage detection, it is assumed that the stiffness of the elements uniformly diminishes with damage occurrence. Therefore, the flexural rigidity, $\boldsymbol{E I}$, of the elements becomes $(1-\boldsymbol{D} \boldsymbol{I}) \times \boldsymbol{E} \boldsymbol{I}$ after damage occurrence. Where $\boldsymbol{D} \boldsymbol{I}$ is the damage index vector of the finite elements as shown in Eq. (32). DI consists of elements with values between 0 and $1 . \boldsymbol{D I}=0$ means that the element is healthy and no 
reduction in modules of elasticity has been observed, and $\boldsymbol{D I}=1$ states that the element is totally damaged with $100 \%$ reduction in modules of elasticity.

$D I=\frac{E_{0}-E_{d}}{E_{0}}$

To evaluate the accuracy of the model, damage detection error is calculated using Eq. (33), where $\boldsymbol{D} \boldsymbol{I}_{\text {iden }}$ and $\boldsymbol{D I}_{\text {real }}$ are identified and real vectors of damage index, respectively.

Detection Error $=\frac{\boldsymbol{D I}_{\text {iden }}-\boldsymbol{D I}_{\text {real }}}{\boldsymbol{D} \boldsymbol{I}_{\text {real }}} \times 100$

Critical speed of the beam is calculated using Eq. (34) and used to obtain speed ratio of the train, where $L$ is the total length of the beam and $T_{1}$ is the first natural period of the beam which is obtainable from the solution to Eq. (14). Mass ratio of the train is also calculated using Eq. (36):

Critical Speed $=\frac{L}{\left(\frac{T_{1}}{2}\right)}(\mathrm{mm} / \mathrm{sec})$,

Speed Ratio $=\frac{\text { Spped of Train }}{\text { Critical Speed }}$

Mass Ratio $=\frac{\text { Average Weight of Moving Axles }}{\text { Weight of longest span of beam }}$.

To simulate the condition of a real test, the measured responses are numerically perturbed to consider the presence of measurement errors. A white noise is added to the calculated responses to simulate the polluted measurements as follows:

$\ddot{\boldsymbol{u}}_{\boldsymbol{m}}=\ddot{\boldsymbol{u}}_{c a l}+e_{p}$ norm $\operatorname{var}\left(\ddot{\boldsymbol{u}}_{c a l}\right)$,

where, $\ddot{\boldsymbol{u}}_{\boldsymbol{m}}$ denotes polluted acceleration response; $e_{p}$ is the noise level (for example $5 \%$ or $10 \%$ ); norm is a standard normal distribution vector with zero mean and unit standard deviation; var(.) is the variance of the time history of calculated acceleration response $\ddot{\boldsymbol{u}}_{\text {cal }}$.

\section{Numerical results}

In this research project a three span beam with 21 finite elements is investigated under multiple number of sprungmasses passing over the beam with a constant speed. Considering 21 finite elements each of which has two degrees of freedom (Dof) in each node and 8 moving axles, the total number of DoFs of the bridge-vehicle system will be 60. As illustrated in Fig. 3, in each span one sensor is located at transitional Dofs close to the mid-span to record the vibration response of the bridge.

Characteristics of the vehicle and bridge model are presented in Table 1, where $N_{e}$ and $N_{v}$ are the number of finite elements and moving axles, respectively. Lel is the length of elements, $\zeta$ denotes modal damping ratio, $I$ is the second moment of inertia of the beam, Vratio and Mratio are speed and mass ratio, respectively as introduced in Eqs. (35-36). Suspension properties of train are assumed based on recent proposed values [23]. Moving vehicle is modelled as a series of 8 sprung masses. As depicted in Fig. 1 the lower mass related to wheel load, $\left(m_{w}\right)$, is supposed to be a known and constant value, but the upper masses representing the vehicle loads, $\left(m_{v}\right)$, have unknown values which need to be identified by the proposed method. The bridge is modelled as an Euler-Bernouli beam with a constant cross sectional area as presented in Fig. 3. The tolerance for damage detection process is set to be $1 \times 1^{0-4}$.

Table 1 Properties of bridge, train and moving loads

\begin{tabular}{cccc}
\hline \multicolumn{4}{c}{ Bridge Properties } \\
\hline$E(\mathrm{MPa})$ & $I\left(\mathrm{~mm}^{4}\right)$ & Lel $(\mathrm{mm})$ & $\zeta$ \\
25000 & $2.58 \mathrm{e} 10$ & 1500 & 0.05 \\
& \multicolumn{2}{c}{ Train Properties } \\
$N_{v}$ & $m_{w}(\mathrm{~kg})$ & $k_{v}(\mathrm{~N} / \mathrm{mm})$ & $C_{v}(\mathrm{~N} . \mathrm{sec} / \mathrm{mm})$ \\
8 & 2500 & 9120 & 86 \\
& \multicolumn{4}{c}{ Moving Loads $(\mathrm{Kg})$} & \\
$M v 1$ & $M v 2$ & $M v 3$ & $M v 3$ \\
18000 & 20000 & 22500 & 20000 \\
$M v 5$ & $M v 6$ & $M v 7$ & $M v 8$ \\
17500 & 20000 & 22500 & 16000 \\
\hline
\end{tabular}

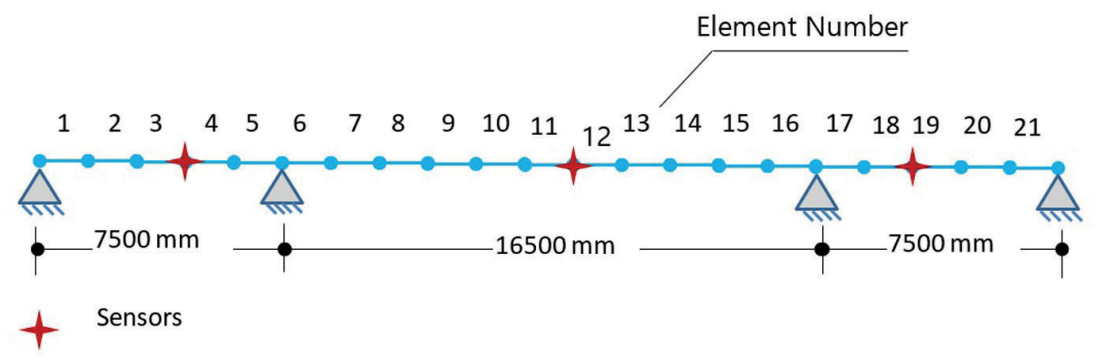

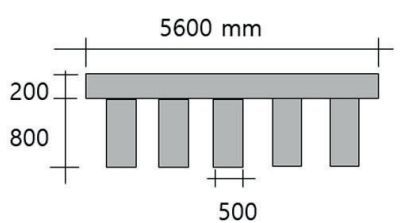

Beam Cross Section

Fig. 3 Structural properties of beam and location of sensors 
Table 2 Multiple damage scenarios

\begin{tabular}{lc}
\hline Scenario & $\begin{array}{c}\text { Element Number } \\
\text { Reduction in Modules of Elasticity (\%) }\end{array}$ \\
\hline I & $4,12,13,19$ \\
& $7 \%, 15 \%, 11 \%, 5 \%$ \\
II & Random (7 out of 21 elements $)$ \\
& Random $(0-20 \%)$ \\
\hline
\end{tabular}

As shown in Table 2, two damage scenarios are applied to the model to represent the capability of the proposed method for identification of structural damages under unknown moving load. In Scenario I, only three elements are considered as damaged with a reduction in elastic modulus, but in scenario II, a random selection of 7 elements with a random reduction in elastic modulus is considered for identification.
After applying the proposed method for Scenario I, Real and identified $\{D I\}$ for beam elements are illustrated in Fig. 4. The proposed method accurately identified all 4 damaged elements, as presented in Fig. 4(a). In this case the identification error using Eq. (33) is $0.97 \%$ and no other healthy elements are detected as damaged. On the other hand, when the measured data are polluted with $5 \%$ and $10 \%$ noise, identification error increases to $2.49 \%$ and $6.95 \%$, respectively. As depicted in Figs. 4(b, c), in the presence of $5 \%$ noise, the proposed method has still its efficiency, while in the case of $10 \%$ some healthy elements are detected as damaged. Although these false detections are minor damages, but they adversely affect total accuracy of the proposed method.

Fig. 5 represents the results of the proposed method for damage scenario II, where 7 elements were selected randomly as damaged with a reduction percentage in modules
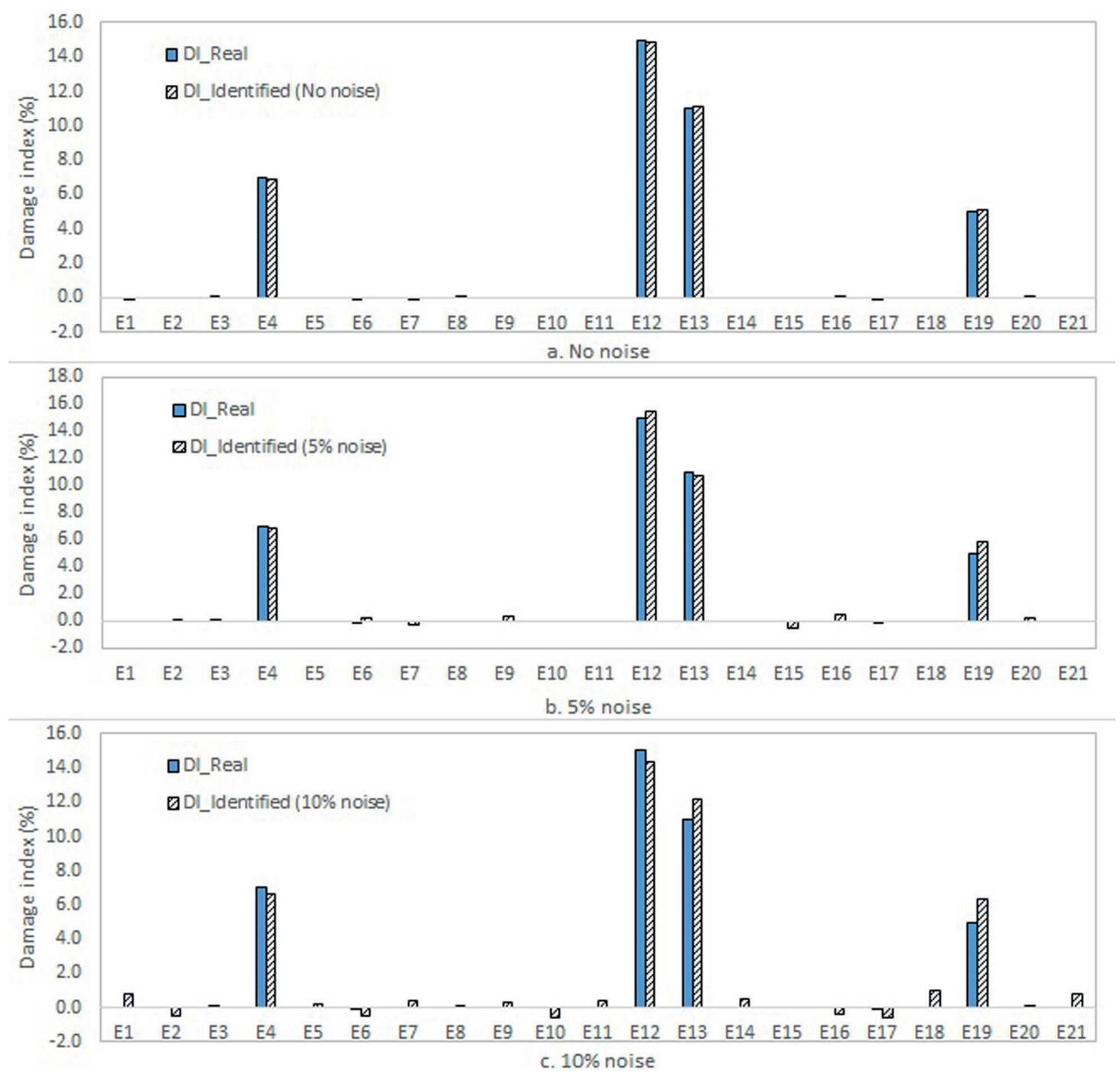

Fig. 4 Real and identified damage indexes $\{D I\}$ for Scenario I in the presence of measurement noise 

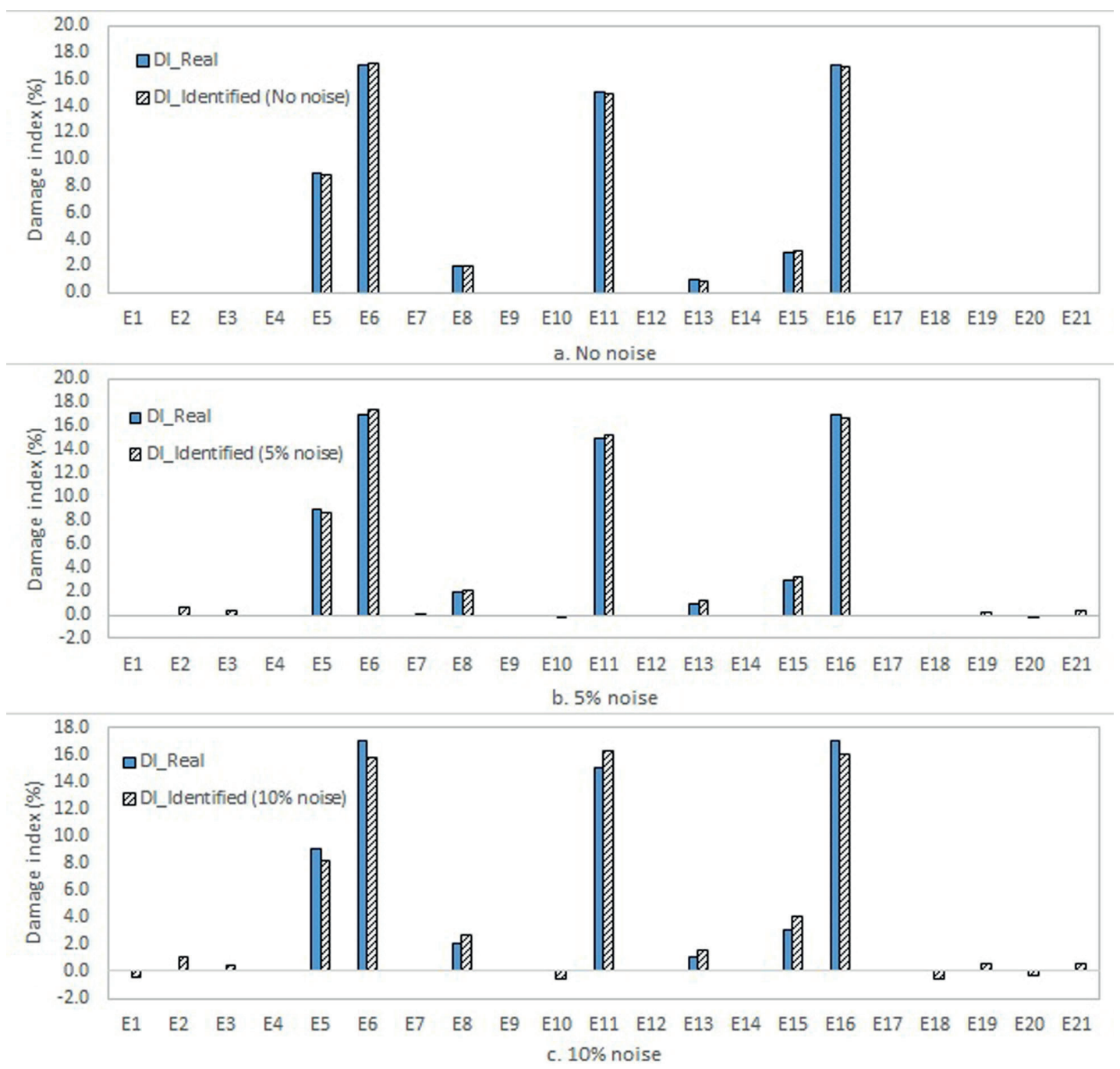

Fig. 5 Real and identified damage indexes $\{D I\}$ for Scenario II in the presence of measurement noise

of elasticity between 5 to $20 \%$. As presented in Fig. 5(a), all damaged elements are identified accurately. In this case elements 5, 6, 8, 11, 13, 15 and 16 are identified as damaged with 9, 17, 2, 15, 1,3 and 17 percent reduction in modules of elasticity, respectively with the identification error of $1.1 \%$. In addition, as illustrated in Figs. 5(b, c), when $5 \%$ and $10 \%$ noise is added to the measured data, the identification error reaches $2.79 \%$ and $7.19 \%$ respectively. This reveals that the proposed method is insensitive to noise when it comes to low levels of measurement noise (noise $<5 \%$ ) and has still its efficiency, while for high levels of measurement noise (noise $>5 \%$ ) using de-noising techniques such as Kalman filter will help the method for separating the real vibrational signal and noise. In this problem, comparing natural frequencies of the damaged and undamaged models revealed that, variations of the first and last 5 natural frequencies of the bridge are $0.4 \%$ and $1.5 \%$ respectively. This demonstrates that modal properties cannot be effectively used as damage indicators in bridge identification problems. In contrast, the acceleration response effectively reflects structural damages even at early stages.

As shown in Fig. 6, the proposed method precisely identifies unknown weight of the 8 moving axles $\left(m_{v}\right)$. The robustness of the present research is the accurate estimation of a set of sprung-masses passing over the beam with known weight.

In the current research, the effect of critical parameters such as mass and speed of the train on accuracy of the identification results is also investigated. Numerical results prove that speed ratio plays a significant role on identification results. Based on the obtained results, when train speed increases and approaches the critical speed, the damage detection error increases dramatically. As shown in Fig. 7(a) selecting speed ratio more than 0.7 will lead to inaccurate results or divergence of the iterative process. On the other hand, selecting speed ratio in the range of 0.5 to 0.7 leads to optimum results which complies with 

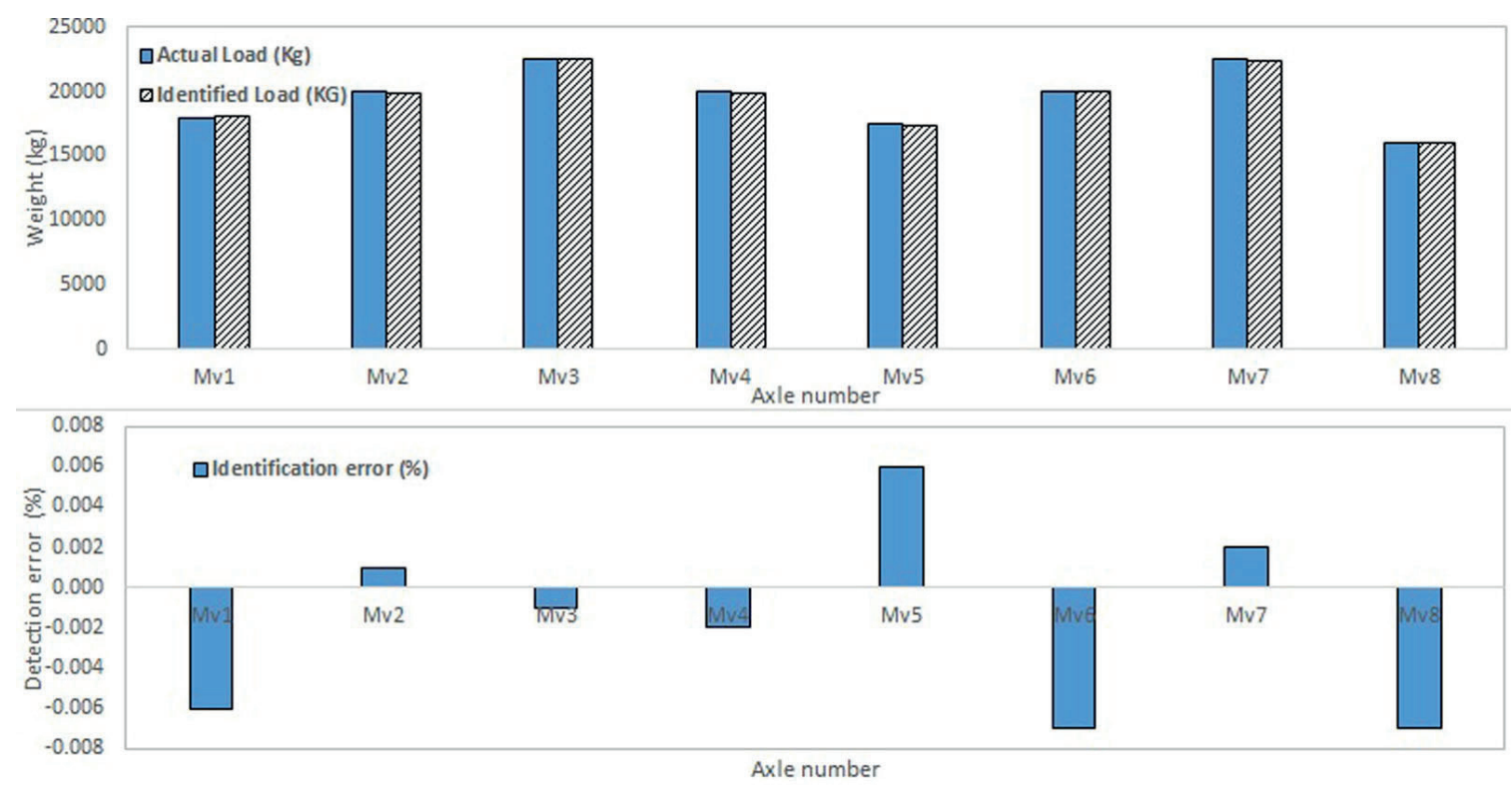

Fig. 6 Moving Load Identification result



(a)

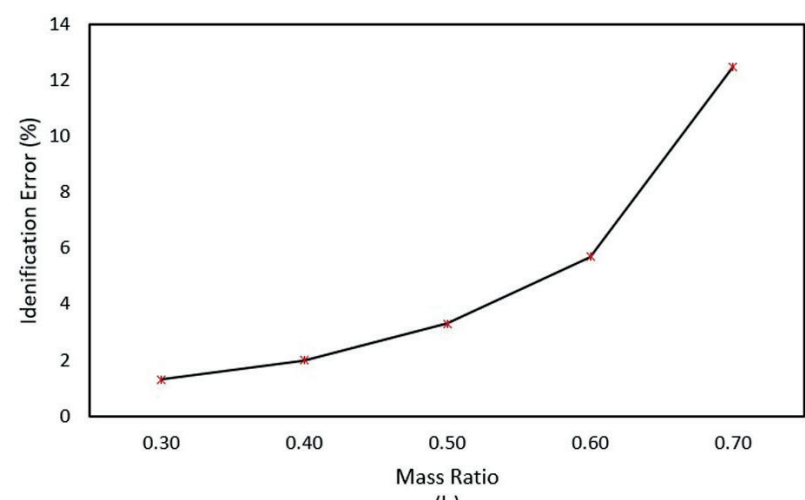

(b)

Fig. 7 Variation of identification error with respect to: (a) speed ratio, (b) mass ratio

the findings of previous researchers [20]. In addition, Fig. 7(b) shows that when the mass ratio of the moving vehicle is relatively high compared to that of the bridge, the nonlinearity effect of the moving loads augments the identification error and causes false damage detection.
Consequently, using mass ratio less than 0.3 and speed ratio between 0.5 to 0.7 is recommended for future works in the same field which leads to optimum results.

\section{Conclusions}

In current study, an improved response sensitivity-based simultaneous identification method is presented for railway bridge structure under the passage of multi-axles sprungmasses with unknown weights. The analyses are conducted in time-domain taking into account the bridge-vehicle interaction. The proposed model-updating procedure is an iterative algorithm which dramatically reduces the computational cost by using sensitivity analysis and Tikhonov regularization method. In order to show the capabilities of the proposed method, a set of numerical examples with multiple damage scenarios are analyzed. In addition, the efficiency of the proposed method for damage identification under the presence of measurement noise is also verified. Numerical results reveal that the proposed method accurately identifies unknown values of the moving loads as well as the location and extent of the structural damages even in the presence of measurement noise. It is also observed that the proposed method is insensitive to low levels of noise (noise $<5 \%$ ). However, for obtaining optimum results for problems with high levels of noise (noise $>5 \%$ ), implementing de-noising techniques on measured data is recommended. After careful consideration of the effect of speed and mass of the moving vehicle on the identification results, an optimal range for these critical parameters is provided. It is also observed that simulating the train in the form of sprung-masses 
guaranties the interaction of bridge-vehicle system and dramatically increases the accuracy of the identification compared to existing research works in this field. Since the proposed method is developed merely based on investigating the recorded time-history of the structural vibration, which takes into account the bridge-vehicle interaction as well as the measurement noise, it can be effectively adopted for application in the real and long-time monitoring of bridge structures under operation.

\section{References}

[1] Doebling, S. W., Farrar, C. R., Prime, M. B., Shevitz, D. W. "Damage Identification and Health Monitoring of Structural and Mechanical Systems from Changes in Their Vibration Characteristics: A Literature Review", Los Alamos National Laboratory, Los Alamos, NM, USA, Rep. LA-13070-MS, 1996.

https://doi.org/10.2172/249299

[2] Ubertini, F., Comanducci, G., Cavalagli, N., Pisello, A. L., Materazzi, A. L., Cotana, F. "Environmental effects on natural frequencies of the San Pietro bell tower in Perugia, Italy, and their removal for structural performance assessment", Mechanical Systems and Signal Processing, 82, pp. 307-322, 2017.

https://doi.org/10.1016/j.ymssp.2016.05.025

[3] Majumder, L., Manohar, C. S. "Nonlinear reduced models for beam damage detection using data on moving oscillator-beam interactions", Computers and Structures, 82(2-3), pp. 301-314, 2004. https://doi.org/10.1016/j.compstruc.2003.08.007

[4] Schommer, S., Nguyen, V. H., Maas, S., Zürbes, A. "Model updating for structural health monitoring using static and dynamic measurements", Procedia Engineering, 199, pp. 2146-2153, 2017. https://doi.org/10.1016/j.proeng.2017.09.156

[5] Feng, D., Feng, M. Q. "Model Updating of Railway Bridge Using In Situ Dynamic Displacement Measurement under Trainloads", Journal of Bridge Engineering, 20(12), Article number: 04015019, 2015 .

https://doi.org/10.1061/(ASCE)BE.1943-5592.0000765

[6] Song, M., Yousefianmoghadam, S., Mohammadi, M.-E., Moaveni, B., Stavridis, A., Wood, R. L. "An application of finite element model updating for damage assessment of a two-story reinforced concrete building and comparison with lidar", Structural Health Monitoring, 17(5), pp. 1129-1150, 2018. https://doi.org/10.1177/1475921717737970

[7] Blachowski, B. "Modal Sensitivity Based Sensor Placement for Damage Identification Under Sparsity Constraint", Periodica Polytechnica Civil Engineering, 63(2), pp. 432-445, 2019. https://doi.org/10.3311/PPci.13888

[8] Feng, D., Sun, H., Feng, M. Q. "Simultaneous identification of bridge structural parameters and vehicle loads", Computers and Structures, 157, pp. 76-88, 2015.

https://doi.org/10.1016/j.compstruc.2015.05.017

[9] Zhang, Q., Jankowski, Ł., Duan, Z. "Simultaneous Identification of Moving Vehicles and Bridge Damages Considering Road Rough Surface", Mathematical Problems in Engineering, Article ID: 963424, 2013.
Obtained results reveal that the proposed method is capable of identifying the location as well as quantifying the different patterns of damages in bridge structure with acceptable accuracy.

\section{Conflict of Interest}

The authors declare that they have no conflict of interest.

[10] Zhao, Y., Noori, M., Altabey, W. A. "Damage detection for a beam under transient excitation via three different algorithms", Structural Engineering and Mechanics", 64(6), pp. 803-817, 2017. https://doi.org/10.12989/sem.2017.64.6.803

[11] Liu, J. K., Wei, Z. T., Lu, Z. R., Ou, Y. J. "Structural damage identification using gravitational search algorithm", Structural Engineering and Mechanics, 60(4), pp. 729-747, 2016. https://doi.org/10.12989/sem.2016.60.4.729

[12] Lu, Z. R., Law, S. S. "Features of dynamic response sensitivity and its application in damage detection", Journal of Sound and Vibration, 303(1-2), pp. 305-329, 2007. https://doi.org/10.1016/j.jsv.2007.01.021

[13] Li, J., Law, S. S., Hao, H. "Improved damage identification in bridge structures subject to moving loads: Numerical and experimental studies", International Journal of Mechanical Sciences, 74, pp. 99-111, 2013.

https://doi.org/10.1016/j.ijmecsci.2013.05.002

[14] Hernandez, E. M. "Identification of isolated structural damage from incomplete spectrum information using 11-norm minimization", Mechanical Systems and Signal Processing, 46(1), pp. 59-69, 2014. https://doi.org/10.1016/j.ymssp.2013.12.009

[15] Zhou, X.-Q., Xia, Y., Weng, S. "L1 regularization approach to structural damage detection using frequency data", Structural Health Monitoring, 14(6), pp. 571-582, 2015. https://doi.org/10.1177/1475921715604386

[16] Ahmadian, H., Mottershead, J. E., Friswell, M. I. "Regularisation methods for finite element model updating", Mechanical Systems and Signal Processing, 12(1), pp. 47-64, 1998. https://doi.org/10.1006/mssp.1996.0133

[17] Li, X. Y., Law, S. S. "Adaptive Tikhonov regularization for damage detection based on nonlinear model updating", Mechanical Systems and Signal Processing, 24(6), pp. 1646-1664, 2010. https://doi.org/10.1016/j.ymssp.2010.02.006

[18] Zheng, T., Liu, J., Luo, W., Lu, Z. "Damage identification using cloud model based fruit fly optimization algorithm", Structural Engineering and Mechanics, 67(3), pp. 245-254, 2018. https://doi.org/10.12989/sem.2018.67.3.245

[19] Huang, J. L., Lu, Z. R. "BB-BC optimization algorithm for structural damage detection using measured acceleration responses", Structural Engineering and Mechanics, 64(3), pp. 353-360, 2017. https://doi.org/10.12989/sem.2017.64.3.353

[20] Fryba, L. "Dynamics of Railway Bridges", Thomas Telford Publishing, London, United Kingdom, 1996. 
[21] Chopra, A. K. "Dynamics of Structures, Theory and Applications to Earthquake Engineering", Prentice Hall, Upper Saddle River, NJ, USA, 2001.

[22] Choi, H. G., Thite, A. N., Thompson, D. J. "Comparison of methods for parameter selection in Tikhonov regularization with application to inverse force determination", Journal of Sound and Vibration, 304(3-5), pp. 894-917, 2017.

https://doi.org/10.1016/j.jsv.2007.03.040
[23] Mu, D., Choi, D.-H. "Dynamic responses of a continuous beam railway bridge under moving high speed train with random track irregularity", International Journal of Steel Structures, 14, pp. 797-810, 2014.

https://doi.org/10.1007/s13296-014-1211-1 